\title{
The association between neutrophil-to-albumin ratio and mortality in patients with cardiogenic shock: a cohort study
}

\section{Yangpei Peng}

Wenzhou Medical University Second Affiliated Hospital https://orcid.org/0000-0002-8122-3374

\section{Yangjing Xue}

Wenzhou Medical University Second Affiliated Hospital

Jinsheng Wang

Wenzhou Medical University Second Affiliated Hospital

Huaqiang Xiang

Wenzhou Medical University Second Affiliated Hospital

Kangting Ji

Wenzhou Medical University Second Affiliated Hospital

Jie Wang ( $\mathbf{3 9 3 5 5 1 0 6 2 @ q q . c o m ) ~}$

https://orcid.org/0000-0003-3357-5743

Cong Lin

Wenzhou Medical University Second Affiliated Hospital

\section{Research article}

Keywords: Neutrophil-to-albumin ratio, Cardiogenic shock, Intensive care unit

Posted Date: October 14th, 2019

DOI: https://doi.org/10.21203/rs.2.16002/v1

License: (c) (i) This work is licensed under a Creative Commons Attribution 4.0 International License.

Read Full License 


\section{Abstract}

Background Cardiogenic shock (CS) is a lethal complication. Given the poor outcome of CS, we performed a retrospective cohort study to identify whether the neutrophil-to-albumin ratio (NAR) was significantly associated with mortality from CS. Methods All patient data were extracted from the MIMIC III version 1.3. Comparisons between groups was made using the chi-square or Fisher's exact tests for categorical variables and the variance analysis or the Kruskal-Wallis test was used for continuous variables. The primary outcome was 30-day mortality and the secondary ones were 90-day and 365-day mortality. We used Cox proportional hazards models to evaluate the association between the various categories of NAR and survival. To further identify the association, subgroup analyses were performed. Results A total of 475 patients with CS were enrolled. A significant positive correlation between NAR levels and 30-day, 90-day or 365-day mortality was observed. For the primary outcome of 30-day mortality, the HR $(95 \% \mathrm{Cl})$ values given NAR levels $23.54-27.86$ and $>27.86$ were $1.72(1.17,2.53)$ and $1.96(1.34,2.87)$ compared with the reference (NAR < 23.47) in tertile analysis. In multivariate analyses, the HR $(95 \% \mathrm{Cl})$ values were still of statistical significance[1.98 $(1.25,3.15)$ and $2.03(1.26,3.26)]$. When quintiles were applied to grouping patients according to NAR level, similar associations were also observed. For the secondary outcomes, the upward trend remained statistically significant. Conclusions NAR level was associated with survival from CS. NAR appeared to be an independent and readily-available prognostic biomarker of mortality in patients with CS.

\section{Background}

Cardiogenic shock (CS), a lethal complication of cardiac emergencies, is traditionally thought to begin with depression of myocardial contractility, followed by intractable hypotension, coronary insufficiency and further loss of cardiac output, causing multiple organ failure and eventually death ${ }^{1,2}$. For decades, the prevalence of CS has risen from $4.1 \%$ to $7.7 \%$ of all admissions to the intensive care unit (ICU) ${ }^{3}$, of which approximately $6.4 \%$ to $40 \%$ were reported to have died despite intensive care ${ }^{4,5}$. Although less frequent than other fatal diseases, CS has had even higher mortality rates and poorer short-term survival, remaining a clinical challenge for medical personnel throughout the world ${ }^{6-8}$.

Previous studies have shown that a systemic inflammatory response, release of cytokines, activation of complement, expression of inducible nitric oxide synthase and improper vasodilatation might exert an influence both on the occurrence and the outcome of shock ${ }^{9}$. Further evidence suggested that inflammation played an important role in the pathogenesis and outcome of CS, a devastating complication of cardiovascular disease ${ }^{10}$. Among the inflammatory mediators, the neutrophil is wellknown as a marker of inflammation ${ }^{11}$. Several studies indicated that low serum albumin levels were associated with high mortality because of all-cause or cardiovascular mortality 12,13 .

Based on these studies, we put forward a hypothesis that neutrophil-to-albumin ratio (NAR) would be associated with mortality from CS. To our knowledge, to date, no study has explored the prognostic 
significance of NAR in patients with CS. Therefore, we performed a retrospective cohort study to identify the associations between NAR and mortality in patients with CS so as to assess prognosis.

\section{Methods}

\subsection{Data source}

All data in our study were extracted from the Medical Information Mart for Intensive Care Database III version 1.3 (MIMIC-III v1.3), a large, open, free and single-centered database including information from more than 50,000 adult patients admitted to various critical care units at Beth Israel Deaconess Medical Center (Boston, MA, USA) from 2001 to $2012^{14}$. The setting and use of this database were approved by the institutional review boards of the Massachusetts Institute of Technology (Boston, MA) and Beth Israel Deaconess Medical Center (Cambridge, MA). All personal information included in the database have been de-identified to safeguard privacy.

\subsection{Population selection criteria}

More than 50,000 ICU admissions to the MIMIC-III database were recorded, and only patients diagnosed with CS were extracted. Among these patients, we selected those who attained more than 16 years of age at first admission while remaining in the hospital for more than 48 hours. Exclusion criteria were as follows: (1) patients who were diagnosed with hematologic neoplasms, including leukemia, multiple myeloma, and others; (2) more than $10 \%$ individual data were missing; (3) individual data values exceeded the mean \pm 3 times the standard deviation (SD).

CS was determined on the grounds of International Classification of Diseases, Ninth Revision (ICD-9), the definitions in the clinical practice guidelines including the ESC Guidelines ${ }^{15}$ as well as clinical trials, including SHOCK ${ }^{16}$ and IABP-SHOCK II ${ }^{17}$.

\subsection{Date extraction}

Data were extracted through Structured Query Language (SQL) ${ }^{18}$ with MySQL tools (version 5.6.24) from MIMIC-III. The extracted data contain demographic parameters, basic vital signs, laboratory indicators and scoring systems.

Demographic parameters included age, gender and ethnicity, while basic vital signs included heart rate $(\mathrm{HR})$, systolic blood pressure (SBP), diastolic blood pressure (DBP), mean blood pressure (MBP), respiratory rate $(\mathrm{RR})$, temperature and percutaneous oxygen saturation $\left(\mathrm{SPO}_{2}\right)$. The following laboratory indicators were extracted: neutrophils, albumin, platelets, partial thromboplastin time (PTT), prothrombin time (PT), international normalized ratio (INR), serum bicarbonate, serum sodium levels, serum potassium levels and serum glucose levels. We defined the NAR as the ratio of the neutrophil percentage to the serum albumin level. We additionally extracted relevant comorbidities, for example, coronary heart disease (CAD), congestive heart failure (CHF), atrial fibrillation (AF), stroke, chronic obstructive pulmonary 
disease (COPD), pneumonia, acute respiratory distress syndrome (ARDS) and other diseases listed in Table 1. Severity-of-illness scores, including the Sequential Organ Failure Assessment (SOFA) ${ }^{19}$ score and the Simplified Acute Physiology Score II (SAPS II) ${ }^{20}$ were also calculated for every individual. The SOFA score, designed to describe a sequence of complications in the critically ill, is the sum of points obtained from evaluation of respiration, coagulation, liver, cardiovascular system, central nervous system and kidney. The SAPS II, a scoring system developed to estimate the risk of death, included 12 physiology variables (HR, SBP, body temperature, partial pressure of arterial oxygen or fraction of inspired oxygen ratio, urinary output, serum urea or serum urea nitrogen level, WBC count, serum potassium level, serum sodium level, serum bicarbonate level, bilirubin level and Glasgow Coma Score ${ }^{21}$ ), age, type of admission (scheduled surgical, unscheduled surgical or medical) and three underlying disease variables (acquired immunodeficiency syndrome, metastatic cancer and hematologic malignancy). All scores were assessed and calculated on the basis of published recommendations and accepted formulas.

The initiation of our study was the time when the patient admitted to ICU. The outcomes were 30-day, 90day and 365-day mortality, in which 30-day mortality was the primary outcome. Baseline characteristics were all recorded within 24 hours after admission to the ICU.

\subsection{Statistical analysis and modeling strategy}

Baseline characteristics delaminated by NAR were presented in Table 1. Categorical data were shown as frequency (percent), while continuous ones as mean (SD) or median(IQR). We did comparisons between groups by the chi-square test ${ }^{22}$ or Fisher's exact test ${ }^{23}$ for categorical variables and the variance analysis or the Kruskal-Wallis test ${ }^{24}$ for continuous ones.

To examine the associations between NAR and outcomes (30-day, 90-day and 365-day mortality), we used Cox proportional hazards models ${ }^{25}$. The outcomes were respectively analysed according to the tertiles or the quintiles of the NAR level. The first tertile or quartile group was regarded as the reference group. The results were presented as hazard ratios (HRs) with $95 \%$ confidence intervals (Cls). To further identify the association between NAR and mortality, multivariate analyses were performed using two adjusted models. The confounders selected in our models were based on their associations with mortality or a mutation exceeding $10 \%{ }^{26}$. In model I, we adjusted covariates for age, gender and ethnicity. In model II, covariates were adjusted further for length of stay in ICU, HR, SBP, DBP, RR, SPO anion gap, serum bicarbonate, serum potassium, $\mathrm{SCr}$, BUN, hematocrit, platelet count, WBC count, PTT, PT, INR, stroke, pneumonia, COPD, chronic liver disease, chronic renal disease, RRT, malignancy, vasoactive agent, SOFA score and SAPSII score. Building further on this foundation, we performed stratified analysis to confirm whether the effect of NAR differed across each of the subgroups that were classified by stay in ICU, vital signs ( $\mathrm{HR}, \mathrm{SBP}, \mathrm{DBP}, \mathrm{RR}$, temperature, $\mathrm{SPO}_{2}$ ), laboratory parameters (anion gap, serum bicarbonate, serum sodium, serum potassium, serum chloride, serum bilirubin, serum glucose, SCr, BUN, hematocrit, hemoglobin, platelet count, WBC count, PTT, PT, INR), comorbidities (CAD, CHF, 
stroke, pneumonia, COPD, respiratory failure, chronic liver disease, chronic renal disease, RRT, malignancy), vasoactive drug use and scoring systems (SOFA and SAPSII scores).

A two-tailed $P$ value $<0.05$ was deemed statistically significant. EmpowerStats version 2.17.8 (http://www.empowerstats.com/cn/) and R software version 3.42 were used for all statistical analysis.

\section{Results}

\subsection{Subject characteristics}

Patient records from more than 50,000 subjects treated in the ICU at Beth Israel Deaconess Medical Center were originally extracted from the MIMIC-III database. After subjects who did not meet the inclusion criteria were excluded, a total of 475 patients with CS remained. As shown in Table 1, among the patients included, 272 (57.3\%) were men and 328 (69.1\%) were white.

All patients included in the study were equally divided into three groups according to NAR: 158 patients were in the low-NAR group (<23.47), 158 patients were in the mid-NAR group (23.54-27.86), and 159 patients were in the high-NAR group (> 27.86). Patients in the higher NAR group $(34.1 \pm 8.0)$ tended to be white and had higher serum chloride, BUN, WBC count, PTT, PT, INR and lower serum bicarbonate, hematocrit, hemoglobin. Participants with higher NAR also had a higher SOFA and SAPSII scores than did those with lower NAR (<23.47). These patients, however, had no apparent differences in age, gender, vital signs, vasoactive drug use or comorbidities listed in the Table 1.

\subsection{NAR levels and mortality}

A total of 180, 218 and 264 deaths were recorded in the 30-day, 90-day and 365-day follow-up periods, respectively. As shown in Table 2, we observed a positive relationship between NAR and mortality from CS.

For the primary outcome of 30-day mortality, we observed that higher NAR was associated with increased risk of mortality in tertile analysis and the HR values of the three models were all significant compared to the reference group (NAR < 23.47). The HR $(95 \% \mathrm{Cl})$ values of the mid tertile (NAR 23.54-27.86) and the upper tertile $(\mathrm{NAR}>27.86)$ were $1.72(1.17,2.53)$ and $1.96(1.34,2.87)$, respectively, when compared with the reference of lower tertile (NAR < 23.47). When adjusted for age, gender and ethnicity in model I, an increasing trend was also observed and the adjusted $\mathrm{HR}(95 \% \mathrm{Cl})$ values for 30-day mortality given NAR of $23.54-27.86$ and $>27.86$ were $1.63(1.10,2.40)$ and $1.99(1.36,2.92)$, respectively. After further adjustment for potential confounders, including HR, SBP, DBP and RR in model II, the upward trend remained statistically significant and the upper tertile (NAR > 27.86), even revealing the highest HR (95\% Cl) of $2.03(1.26,3.26)$. 
When stratifying the NAR levels into quintiles and defining the extremely low $(N A R<21.74)$ NAR as a reference, we further confirmed the upward trend between NAR levels and 30-day mortality ( $P$ trend 0.0034 in model II). However, the HRs of the second quintile (NAR 21.76 - 24.29) in the three models and the third quintile (NAR 24.35 - 26.91) in model I didn't have statistical significance compared to the reference group (NAR < 21.74).

Furthermore, the similar trends were also observed for the secondary outcomes of 90-day and 365-day mortality. In tertile analysis, the adjusted HR (95\% Cl) values for 90-day mortality given NAR of 23.5427.86 and $>27.86$ were $1.71(1.14,2.55)$ and $1.93(1.27,2.93)$ in model II, while those for 365 -day mortality were $1.93(1.34,2.77)$ and $2.36(1.61,3.47)$. In quintile analysis, the upward trend between NAR and 90-day or 365-day mortality continued to make sense. The HRs of the second quintile (NAR 21.7624.29), however, also showed no statistical significance.

The results are presented in Table 2 and Figures 1 and 2.

\subsection{Subgroup analyses}

Subgroup analyses were conducted to determine the consistency of association between NAR and 90day mortality in patients with CS (Table 3).

Most subgroup factors showed weak significance with 90-day mortality, except for the length of stay in ICU $(p=0.0465)$, the serum sodium $(p=0.0270)$, the serum bilirubin $(p=0.0343)$, respiratory failure $(p=$ $0.0102)$ and RRT ( $p=0.0044)$. NAR particularly showed significant interactions in patients without RRT. Patients without the therapy of RRT had a significant higher 90- day mortality risk for NAR $>27.86$ [HR $(95 \% \mathrm{Cl}) 2.29(1.58,3.32)]$. While patients without respiratory failure had higher risks with NAR $>27.86$ [HR $(95 \% \mathrm{Cl}) 3.14(1.89,5.20)$ vs HR $(95 \% \mathrm{Cl}) 1.19(0.75,1.90)]$ and NAR $23.54-27.86$ [HR (95\% Cl) 1.73 $(1.02,2.95)$ vs. HR $(95 \% \mathrm{Cl}) 1.22(0.76,1.94)]$. In the subgroups of the length of stay in ICU, the serum sodium and the serum bilirubin, however, the interactions were of relatively weak significance. All of the subgroup analyses were demonstrated in Table 3.

\section{Discussion}

In our study, we observed a significant positive correlation between NAR levels and 30-day, 90-day or 365day mortality in patients with CS. In particular a high level of NAR was associated with growing risk of mortality. After adjusting for potential confounders, the differences in each outcome retained statistical significance.

CS is a lethal complication of cardiovascular diseases with an extremely high mortality. Inflammation has been shown to play a vital role in the pathogenesis and outcome of CS. Studies in recent decades have suggested the prognostic value of inflammatory mediators in CS, including blood cells ${ }^{27}$, cytokines $^{28,29}$, complement ${ }^{30}$ and enzymes ${ }^{31,32}$. Furthermore, the use of albumin, the main serum protein, to predict the mortality of cardiovascular disease as well as all-cause mortality has already been 
described ${ }^{12,33,34}$. As blood cells and serum albumin can directly indicate the host environment, recent studies have combined them, just as the NAR, to predict the outcome of diseases. The NAR, a combination of the neutrophil percentage and the serum albumin level, is a novel and readily-available biomarker. Prior to our work, the prognostic value of NAR was recently shown. Samuel et al. ${ }^{35}$ demonstrated that NAR was a significant prognostic hallmark in patients with palliative pancreatic cancer. Tawfik et al. ${ }^{36}$ investigated the association between NAR and pathological complete response in rectal cancer patients after neoadjuvant chemoradiation. Based on this evidence, an inference may be put forward that NAR levels could predict the mortality in patients with CS.

It remains unclear why NAR, the combined biomarker, could have such a significant prognostic value in patients with CS. On the one hand, neutrophils, which are vital leukocytes, have been well-studied regarding the generation and development of various diseases, including CS. Sionis et al. ${ }^{37}$ recently found that distinct microparticles released by neutrophils (CD15+) significantly increased in patients with $\mathrm{CS}$. This result suggested high activation of neutrophils in CS and further indicated the significance of inflammation in that condition. Given the severe systemic inflammatory response in CS, it was demonstrated that patients with higher leukocyte count had a higher mortality in cardiogenic shock ${ }^{38}$. However, whether the increase of inflammatory mediators in CS results from the heart itself, from intestinal bacterial translocation, or from ischemia-reperfusion injury remains unknown ${ }^{10}$. On the other hand, serum albumin, synthesized in the liver, is the major plasma protein in human blood. Albumin has already been used to predict mortality especially in critically ill patients in ICUs ${ }^{39}, 40$, and even has been a part of major risk scores, for example, the Acute Physiology and Chronic Health Evaluation (APACHE) III Prognostic system and the Framingham risk score for cardiovascular risk. Low albumin levels were demonstrated to be related to some inflammatory mediators 41 , 42 ; therefore, the association between serum albumin and mortality may result from subclinical inflammation, as Mutsert et al. ${ }^{43}$ found in their study. However, whether the prognostic value of albumin only reflects inflammation or whether there is an independent role of albumin itself remains to be turned determined. As albumin plays an important role in maintaining the plasma colloid osmotic pressure, low albumin levels may disorganize the fluid distribution in the internal environment so as to destroy the balance of the hemodynamics, resulting in poor outcomes ${ }^{44}$. Another interpretation may involve the state of nutrition because lower albumin is possibly related to malnutrition, emaciation or cachexia ${ }^{45}$. Furthermore, as the most abundant carrier protein in plasma, albumin can change the existing form of some toxins by binding to them, leading to changes in their biological effects. The recent study of Watanabe et al. ${ }^{46}$ indicated that, when combined with lower albumin levels, levels of indoxyl sulfate, a protein-bound uremic toxin, might be a prognostic marker for cardiovascular diseases, because lower albumin levels might increase free indoxyl sulfate levels, possibly activating a signal transduction pathway and subsequently exerting toxic effects. Further studies are needed to confirm these hypotheses.

CS in critically ill patients has an extremely high mortality. This poor outcome may be affected by a number of factors, including basic vital signs (i.e., $\mathrm{DBP}^{47}, \mathrm{MBP}^{48}$ ), some laboratory parameters (i.e., 
serum bicarbonate levels $\left.{ }^{49}\right)$, cardiac power index ${ }^{48}$, vasopressor support ${ }^{50}$, severity-of-illness scores (i.e., SAPSII ${ }^{48}$ ), as well as other comorbidities. In subgroup analyses, patients were stratified according to potential confounders and statistically significant interactions were observed for some factors, such as respiratory failure and RRT. Patients without a history of respiratory failure or without the therapy of RRT might have a higher risk of 90- day mortality. In patients with the therapy of RRT, prognosis might be meliorated through metabolites clearance. While in patients with a history of respiratory failure, the improved survival might contribute to the systemic antimicrobial therapy and advanced assisted ventilation strategies. The real mechanism, however, remained unclear.

Our study was the first study to explore the prognostic effect of NAR in patients with CS. The period of follow-up in our study was quite long. To further confirm the significant association between NAR and outcomes, we applied quintiles to grouping patients and performed subgroup analyses. The limitations of this study, however, cannot be ignored. First and foremost, it was a retrospective observational study in a single center. The biases inherent in this type of study and selection bias in this design should be highlighted. Therefore, we should further perform studies based on multiple centers. Second, owing to the relatively low incidence of CS, the sample size of patients selected in our study was small, suggesting that larger prospective studies are needed. Third, the NAR was measured only when patients first admitted to the ICU, possibly causing errors to a certain extent. Therefore, the dynamic evaluation of NAR during the ICU stay can make a difference. Furthermore, merely measuring NAR does not adequately reflect genuine levels of inflammation. Therefore, simultaneous measurement of other inflammatory factors would make a better demonstration of our conclusions. Last but not the least, to set up NAR as a prognostic biomarker, its clinical significance must further be verified.

\section{Conclusions}

We found that NAR level was associated with survival of CS. Furthermore, NAR appeared to be an independent and readily available prognostic biomarker of outcomes in patients with CS. However, further prospective studies with larger sample size are needed to confirm our findings.

\section{Declarations}

\subsection{Ethics approval and consent to participate}

Not applicable.

\subsection{Consent for publication}

Not applicable.

\subsection{Competing interests}


The authors have no competing interests to declare.

\subsection{Funding}

No funding.

\subsection{Authors' contributions}

Yangpei Peng, Jie Wang and Cong Lin conceived and designed the research. Yangjing Xue, Jinsheng Wang and Huaqiang Xiang performed the studies and participated in data collection. Jie Wang, Yangjing Xue and Kangting Ji completed data processing and statistical analysis. Yangpei Peng, Yangjing Xue and Cong Lin drafted the manuscript. All authors read and approved the final manuscript.

\subsection{Acknowledgements}

We acknowledge the support from the Second Affiliated Hospital of Wenzhou Medical University.

\subsection{Availability of data and materials}

All data in our study were extracted from a freely accessible database, the Medical Information Mart for Intensive Care Database III version 1.3 (MIMIC-III v1.3). The setting and use of this database were approved by the institutional review boards of the Massachusetts Institute of Technology (Boston, MA) and Beth Israel Deaconess Medical Center (Cambridge, MA). Data are available from MIMIC-III for researchers who meet the criteria for access to the database.

\section{References}

1. Tewelde SZ, Liu SS, and Winters ME. Cardiogenic Shock. Cardiol. Clin. 2018;36:53-61.

2. van Diepen S, Katz JN, Albert NM, et al. Contemporary Management of Cardiogenic Shock: A Scientific Statement From the American Heart Association. Circulation 2017;136:e232-e268.

3. Puymirat E, Fagon JY, Aegerter P, et al. Cardiogenic shock in intensive care units: evolution of prevalence, patient profile, management and outcomes, 1997-2012. Eur. J. Heart Fail. 2017;19:192200.

4. Azoulay E, Adrie C, De Lassence A, et al. Determinants of postintensive care unit mortality: a prospective multicenter study. Crit. Care Med. 2003;31:428-32.

5. Knaus WA, Wagner DP, Zimmerman JE, et al. Variations in mortality and length of stay in intensive care units. Ann. Intern. Med. 1993;118:753-61.

6. Nguyen HL, Yarzebski J, Lessard D, et al. Ten-Year (2001-2011) Trends in the Incidence Rates and Short-Term Outcomes of Early Versus Late Onset Cardiogenic Shock After Hospitalization for Acute Myocardial Infarction. J Am Heart Assoc 2017;6.

7. Aushev A, Ripoll VR, Vellido A, et al. Feature selection for the accurate prediction of septic and cardiogenic shock ICU mortality in the acute phase. PLoS One 2018;13:e0199089. 
8. Goldberg RJ, Spencer FA, Gore JM, et al. Thirty-year trends (1975 to 2005) in the magnitude of, management of, and hospital death rates associated with cardiogenic shock in patients with acute myocardial infarction: a population-based perspective. Circulation 2009;119:1211-9.

9. Hochman JS. Cardiogenic shock complicating acute myocardial infarction: expanding the paradigm. Circulation 2003;107:2998-3002.

10. Geppert A and Huber K. Inflammation and cardiovascular diseases: lessons that can be learned for the patient with cardiogenic shock in the intensive care unit. Curr. Opin. Crit. Care 2004;10:347-53.

11. Gibson $\mathrm{PH}$, Croal BL, Cuthbertson $\mathrm{BH}$, et al. Preoperative neutrophil-lymphocyte ratio and outcome from coronary artery bypass grafting. Am. Heart J. 2007;154:995-1002.

12. Grimm G, Haslacher $H$, Kampitsch $T$, et al. Sex differences in the association between albumin and all-cause and vascular mortality. Eur. J. Clin. Invest. 2009;39:860-5.

13. Takata Y, Ansai T, Soh I, et al. Serum albumin levels as an independent predictor of 4-year mortality in a community-dwelling 80-year-old population. Aging Clin. Exp. Res. 2010;22:31-5.

14. Johnson AE, Pollard TJ, Shen L, et al. MIMIC-III, a freely accessible critical care database. Sci Data 2016;3:160035.

15. Ponikowski P Fau - Voors AA, Voors Aa Fau - Anker SD, Anker Sd Fau - Bueno H, et al. 2016 ESC Guidelines for the Diagnosis and Treatment of Acute and Chronic Heart Failure.

16. Hochman JS, Sleeper La Fau - Webb JG, Webb Jg Fau - Sanborn TA, et al. Early revascularization in acute myocardial infarction complicated by cardiogenic shock. SHOCK Investigators. Should We Emergently Revascularize Occluded Coronaries for Cardiogenic Shock.

17. Thiele H, Zeymer U Fau - Neumann F-J, Neumann Fj Fau - Ferenc M, et al. Intraaortic balloon support for myocardial infarction with cardiogenic shock.

18. Jamison DC. Structured Query Language (SQL) fundamentals. Curr Protoc Bioinformatics 2003;Chapter 9:Unit9.2.

19. Vincent JL, Moreno R, Takala J, et al. The SOFA (Sepsis-related Organ Failure Assessment) score to describe organ dysfunction/failure. On behalf of the Working Group on Sepsis-Related Problems of the European Society of Intensive Care Medicine. Intensive Care Med. 1996;22:707-10.

20. Le Gall JR, Lemeshow S, and Saulnier F. A new Simplified Acute Physiology Score (SAPS II) based on a European/North American multicenter study. JAMA 1993;270:2957-63.

21. Sternbach GL. The Glasgow coma scale. J. Emerg. Med. 2000;19:67-71.

22. Tallarida RJ and Murray RB, Chi-Square Test, in Manual of Pharmacologic Calculations: With Computer Programs, R.J. Tallarida and R.B. Murray, Editors. 1987, Springer New York: New York, NY. p. $140-142$.

23. Fisher's Exact Test, in Encyclopedia of Genetics, Genomics, Proteomics and Informatics, G.P. Rédei, Editor. 2008, Springer Netherlands: Dordrecht. p. 690-690.

24. Analysis of variance and the Kruskal-Wallis test, in Introductory Statistics with R, P. Dalgaard, Editor. 2002, Springer New York: New York, NY. p. 111-127. 
25. Cox Proportional Hazards Model, in Encyclopedia of Public Health, W. Kirch, Editor. 2008, Springer Netherlands: Dordrecht. p. 176-176.

26. Jaddoe VW, de Jonge LL, Hofman A, et al. First trimester fetal growth restriction and cardiovascular risk factors in school age children: population based cohort study. BMJ 2014;348:g14.

27. Gagnon DR, Zhang TJ, Brand FN, et al. Hematocrit and the risk of cardiovascular disease-the Framingham study: a 34-year follow-up. Am. Heart J. 1994;127:674-82.

28. Prondzinsky $\mathrm{R}$, Unverzagt $\mathrm{S}$, Lemm $\mathrm{H}$, et al. Interleukin- $6,-7,-8$ and -10 predict outcome in acute myocardial infarction complicated by cardiogenic shock. Clin. Res. Cardiol. 2012;101:375-84.

29. Prondzinsky R, Unverzagt S, Lemm H, et al. Acute myocardial infarction and cardiogenic shock: prognostic impact of cytokines: INF-gamma, TNF-alpha, MIP-1beta, G-CSF, and MCP-1 beta. Med Klin Intensivmed Notfmed 2012;107:476-84.

30. Granger CB, Mahaffey KW, Weaver WD, et al. Pexelizumab, an anti-C5 complement antibody, as adjunctive therapy to primary percutaneous coronary intervention in acute myocardial infarction: the COMplement inhibition in Myocardial infarction treated with Angioplasty (COMMA) trial. Circulation 2003;108:1184-90.

31. Dominguez-Rodriguez A, Samimi-Fard S, Abreu-Gonzalez P, et al. Prognostic value of admission myeloperoxidase levels in patients with ST-segment elevation myocardial infarction and cardiogenic shock. Am. J. Cardiol. 2008;101:1537-40.

32. Cotter G, Kaluski E, Milo O, et al. LINCS: L-NAME (a NO synthase inhibitor) in the treatment of refractory cardiogenic shock: a prospective randomized study. Eur. Heart J. 2003;24:1287-95.

33. Goldwasser $P$ and Feldman J. Association of serum albumin and mortality risk. J. Clin. Epidemiol. 1997;50:693-703.

34. Okamura T, Hayakawa T, Kadowaki T, et al. A combination of serum low albumin and above-average cholesterol level was associated with excess mortality. J. Clin. Epidemiol. 2004;57:1188-95.

35. Tingle SJ, Severs GR, Goodfellow M, et al. NARCA: A novel prognostic scoring system using neutrophil-albumin ratio and Ca19-9 to predict overall survival in palliative pancreatic cancer. J. Surg. Oncol. 2018;118:680-686.

36. Tawfik B, Mokdad AA, Patel PM, et al. The neutrophil to albumin ratio as a predictor of pathological complete response in rectal cancer patients following neoadjuvant chemoradiation. Anticancer Drugs 2016;27:879-83.

37. Sionis A, Suades R, Sans-Rosello J, et al. Circulating microparticles are associated with clinical severity of persistent ST-segment elevation myocardial infarction complicated with cardiogenic shock. Int. J. Cardiol. 2018;258:249-256.

38. Barron HV, Cannon CP, Murphy SA, et al. Association between white blood cell count, epicardial blood flow, myocardial perfusion, and clinical outcomes in the setting of acute myocardial infarction: a thrombolysis in myocardial infarction 10 substudy. Circulation 2000;102:2329-34.

39. Gillum RF and Makuc DM. Serum albumin, coronary heart disease, and death. Am. Heart J. 1992;123:507-13. 
40. Weijenberg MP, Feskens EJ, Souverijn JH, et al. Serum albumin, coronary heart disease risk, and mortality in an elderly cohort. Epidemiology 1997;8:87-92.

41. Sullivan DH, Roberson PK, Johnson LE, et al. Association between inflammation-associated cytokines, serum albumins, and mortality in the elderly. J. Am. Med. Dir. Assoc. 2007;8:458-63.

42. Nelson JJ, Liao D, Sharrett AR, et al. Serum albumin level as a predictor of incident coronary heart disease: the Atherosclerosis Risk in Communities (ARIC) study. Am. J. Epidemiol. 2000;151:468-77.

43. de Mutsert R, Grootendorst DC, Indemans F, et al. Association between serum albumin and mortality in dialysis patients is partly explained by inflammation, and not by malnutrition. J. Ren. Nutr. 2009;19:127-35.

44. Rackow EC, Fein IA, and Leppo J. Colloid osmotic pressure as a prognostic indicator of pulmonary edema and mortality in the critically ill. Chest 1977;72:709-13.

45. Evans WJ, Morley JE, Argiles J, et al. Cachexia: a new definition. Clin. Nutr. 2008;27:793-9.

46. Watanabe I, Tatebe J, Fujii T, et al. Prognostic Significance of Serum Indoxyl Sulfate and Albumin for Patients with Cardiovascular Disease. Int. Heart J. 2018.

47. Axler O. Low diastolic blood pressure as best predictor of mortality in cardiogenic shock*. Crit. Care Med. 2013;41:2644-7.

48. Popovic B, Fay R, Cravoisy-Popovic A, et al. Cardiac power index, mean arterial pressure, and Simplified Acute Physiology Score II are strong predictors of survival and response to revascularization in cardiogenic shock. Shock 2014;42:22-6.

49. Wigger O, Bloechlinger S, Berger D, et al. Baseline serum bicarbonate levels independently predict short-term mortality in critically ill patients with ischaemic cardiogenic shock. Eur Heart J Acute Cardiovasc Care 2018;7:45-52.

50. Geppert A, Dorninger A, Delle-Karth G, et al. Plasma concentrations of interleukin-6, organ failure, vasopressor support, and successful coronary revascularization in predicting 30-day mortality of patients with cardiogenic shock complicating acute myocardial infarction. Crit. Care Med. 2006;34:2035-42.

\section{Tables}




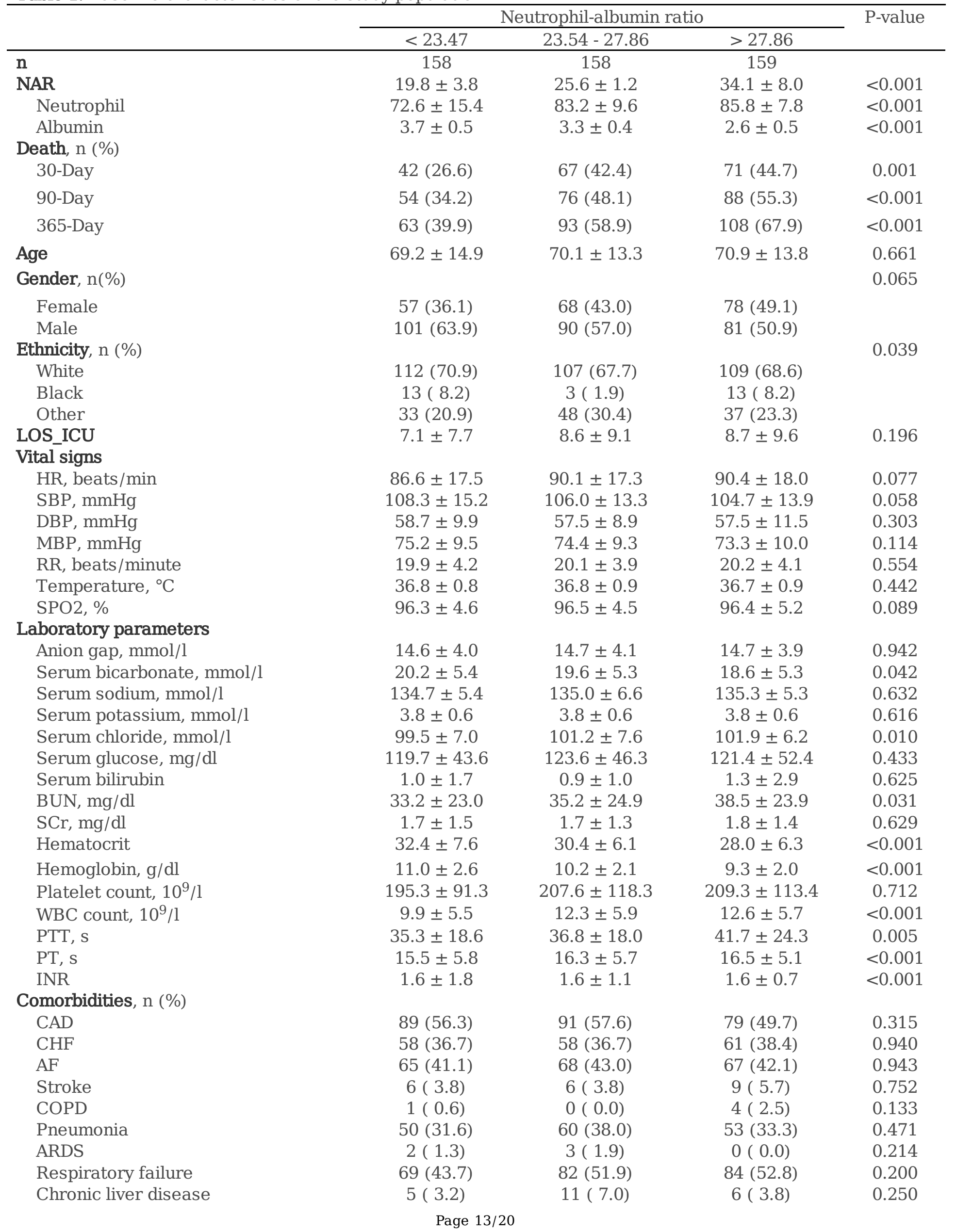


Chronic renal disease

RRT

Malignancy

Vasoactive drug, $\mathrm{n}(\%)$

Scoring systems, mean (Q1-Q3)
30 (19.0)

16 (10.1)

21 (13.3)

$113(71.5)$
28 (17.7)

27 (17.1)

15 ( 9.5)

$126(79.7)$

SOFA

$$
6.7 \pm 3.6
$$

$6.8 \pm 4.0$

$7.9 \pm 3.7$

0.005

$45.5 \pm 15.3$

$52.8 \pm 16.1$

$<0.001$

NAR: neutrophil-to-albumin ratio; N: number; LOS_ICU: length of stay in intensive care unit; HR: heart rate; SBP: systolic blood pressure; DBP: diastolic blood pressure; MBP: mean blood pressure; RR: respiratory rate; SPO2: percutaneous oxygen saturation; BUN: blood urea nitrogen; SCr: Serum creatinine; WBC: white blood cell; PTT: partial thromboplastin time; PT: prothrombin time; INR: international normalized ratio; CAD: coronary heart disease; CHF: congestive heart failure; $\mathrm{AF}$ : atrial fibrillation; COPD: chronic obstructive pulmonary disease; ARDS: acute respiratory distress syndrome; RRT: renal replacement therapy; SOFA: Sequential Organ Failure Assessment; SAPSII: Simplified Acute Physiology Score. Normally distributed data are presented as the mean $\pm \mathrm{SD}$, non-normally distributed data are presented as median (IQR) and categorical variables are presented as $\mathrm{n}(\%)$. 
Table 2. HRs (95\% CIs) for mortality across groups of neutrophil-to-albumin ratio

\begin{tabular}{|c|c|c|c|c|c|c|c|c|c|}
\hline & \multicolumn{3}{|c|}{ Non-adjusted } & \multicolumn{3}{|c|}{ Model I } & \multicolumn{3}{|c|}{ Model II } \\
\hline & $\begin{array}{l}\mathrm{HR} \\
(95 \% \\
\mathrm{CI})\end{array}$ & $\mathrm{P}$ value & $\mathrm{P}$ trend & $\begin{array}{c}\mathrm{HR} \\
(95 \% \\
\mathrm{CI}) \\
\end{array}$ & $P$ value & $\mathrm{P}$ trend & $\begin{array}{c}\mathrm{HR} \\
(95 \% \\
\mathrm{CI}) \\
\end{array}$ & P value & $P$ trend \\
\hline \multirow{2}{*}{\multicolumn{2}{|c|}{ 30-day mortality }} & & \multirow{3}{*}{0.0008} & & & \multirow{3}{*}{0.0005} & & & \multirow{5}{*}{0.0096} \\
\hline & & & & & & & & & \\
\hline 23.47 & 1.0 & & & 1.0 & & & 1.0 & & \\
\hline $\begin{array}{l}23.54 \text { - } \\
27.86\end{array}$ & $\begin{array}{l}1.72 \\
(1.17 \\
2.53)\end{array}$ & 0.0060 & \multirow{7}{*}{0.0001} & $\begin{array}{l}1.63 \\
(1.10 \\
2.40)\end{array}$ & 0.0139 & \multirow{7}{*}{0.0002} & $\begin{array}{l}1.98 \\
(1.25, \\
3.15)\end{array}$ & 0.0036 & \\
\hline $\begin{array}{c}> \\
27.86\end{array}$ & $\begin{array}{l}1.96 \\
(1.34 \\
2.87)\end{array}$ & 0.0006 & & $\begin{array}{l}1.99 \\
(1.36 \\
2.92)\end{array}$ & 0.0004 & & $\begin{array}{l}2.03 \\
(1.26, \\
3.26)\end{array}$ & 0.0036 & \\
\hline $\begin{array}{l}\text { Quintiles } \\
< \\
21.74\end{array}$ & 1.0 & & & 1.0 & & & 1.0 & & \multirow[t]{5}{*}{0.0034} \\
\hline $\begin{array}{l}21.76 \text { - } \\
24.29\end{array}$ & $\begin{array}{l}1.13 \\
(0.65, \\
1.97)\end{array}$ & 0.6524 & & $\begin{array}{l}1.01 \\
(0.58 \\
1.76)\end{array}$ & 0.9695 & & $\begin{array}{l}1.21 \\
(0.64, \\
2.29)\end{array}$ & 0.5632 & \\
\hline $\begin{array}{l}24.35 \text { - } \\
26.91\end{array}$ & $\begin{array}{l}1.82 \\
(1.10 \\
3.02)\end{array}$ & 0.0201 & & $\begin{array}{l}1.63 \\
(0.98 \\
2.71)\end{array}$ & 0.0610 & & $\begin{array}{l}2.08 \\
(1.14, \\
3.80)\end{array}$ & 0.0170 & \\
\hline $\begin{array}{l}26.97 \text { - } \\
30.65\end{array}$ & $\begin{array}{l}2.06 \\
(1.25, \\
3.38)\end{array}$ & 0.0045 & & $\begin{array}{l}2.12 \\
(1.29 \\
3.49)\end{array}$ & 0.0031 & & $\begin{array}{l}2.17 \\
(1.21, \\
3.91)\end{array}$ & 0.0094 & \\
\hline$>30.67$ & $\begin{array}{l}2.29 \\
(1.39 \\
3.76)\end{array}$ & 0.0011 & & $\begin{array}{l}2.11 \\
(1.29 \\
3.47)\end{array}$ & 0.0032 & & $\begin{array}{l}2.36 \\
(1.29, \\
4.30)\end{array}$ & 0.0051 & \\
\hline \multirow{2}{*}{\multicolumn{2}{|c|}{$\begin{array}{l}\text { 90-day mortality } \\
\text { Tertiles }\end{array}$}} & & \multirow{3}{*}{0.0001} & & & & & & \multirow{5}{*}{0.0037} \\
\hline & & & & & & \multirow{4}{*}{$<0.0001$} & & & \\
\hline $\begin{array}{c}< \\
23.47\end{array}$ & 1.0 & & & 1.0 & & & 1.0 & & \\
\hline $\begin{array}{l}23.54 \text { - } \\
27.86\end{array}$ & $\begin{array}{l}1.56 \\
(1.10 \\
2.22)\end{array}$ & 0.0122 & & $\begin{array}{l}1.50 \\
(1.06, \\
2.13)\end{array}$ & 0.0229 & & $\begin{array}{l}1.71 \\
(1.14, \\
2.55)\end{array}$ & 0.0092 & \\
\hline $\begin{array}{c}> \\
27.86\end{array}$ & $\begin{array}{l}1.95 \\
(1.39 \\
2.73)\end{array}$ & 0.0001 & & $\begin{array}{l}1.99 \\
(1.42 \\
2.80)\end{array}$ & $<0.0001$ & & $\begin{array}{l}1.93 \\
(1.27, \\
2.93)\end{array}$ & 0.0022 & \\
\hline Quintiles & 1.0 & & \multirow[t]{5}{*}{$<0.0001$} & 1.0 & & \multirow[t]{5}{*}{$<0.0001$} & 1.0 & & \multirow[t]{5}{*}{0.0010} \\
\hline $\begin{array}{c}21.76- \\
24.29\end{array}$ & $\begin{array}{l}1.15 \\
(0.71 \\
1.86)\end{array}$ & 0.5825 & & $\begin{array}{l}1.03 \\
(0.63 \\
1.67)\end{array}$ & 0.9152 & & $\begin{array}{l}1.17 \\
(0.68, \\
2.02)\end{array}$ & 0.5771 & \\
\hline $\begin{array}{l}24.35 \text { - } \\
26.91\end{array}$ & $\begin{array}{l}1.60 \\
(1.01 \\
2.54)\end{array}$ & 0.0447 & & $\begin{array}{l}1.46 \\
(0.92 \\
2.32)\end{array}$ & 0.1074 & & $\begin{array}{l}1.75 \\
(1.03, \\
2.97)\end{array}$ & 0.0402 & \\
\hline $\begin{array}{l}26.97 \text { - } \\
30.65\end{array}$ & $\begin{array}{l}1.97 \\
(1.26, \\
3.07)\end{array}$ & 0.0028 & & $\begin{array}{l}2.03 \\
(1.30 \\
3.17)\end{array}$ & 0.0019 & & $\begin{array}{l}1.94 \\
(1.17, \\
3.24)\end{array}$ & 0.0107 & \\
\hline$>30.67$ & $\begin{array}{l}2.28 \\
(1.47 \\
3.54)\end{array}$ & 0.0002 & & $\begin{array}{l}2.12 \\
(1.37 \\
3.30)\end{array}$ & 0.0008 & & $\begin{array}{l}2.26 \\
(1.33, \\
3.86)\end{array}$ & 0.0026 & \\
\hline \multicolumn{2}{|c|}{ 365-day mortality } & & \multirow{3}{*}{$<0.0001$} & & & \multirow{3}{*}{$<0.0001$} & & & \multirow{3}{*}{$<0.0001$} \\
\hline $\begin{array}{l}\text { Tertiles } \\
< \\
23.47\end{array}$ & 1.0 & & & 1.0 & & & 1.0 & & \\
\hline $23.54-$ & 1.69 & 0.0013 & & 1.65 & 0.0022 & & 1.93 & 0.0004 & \\
\hline
\end{tabular}




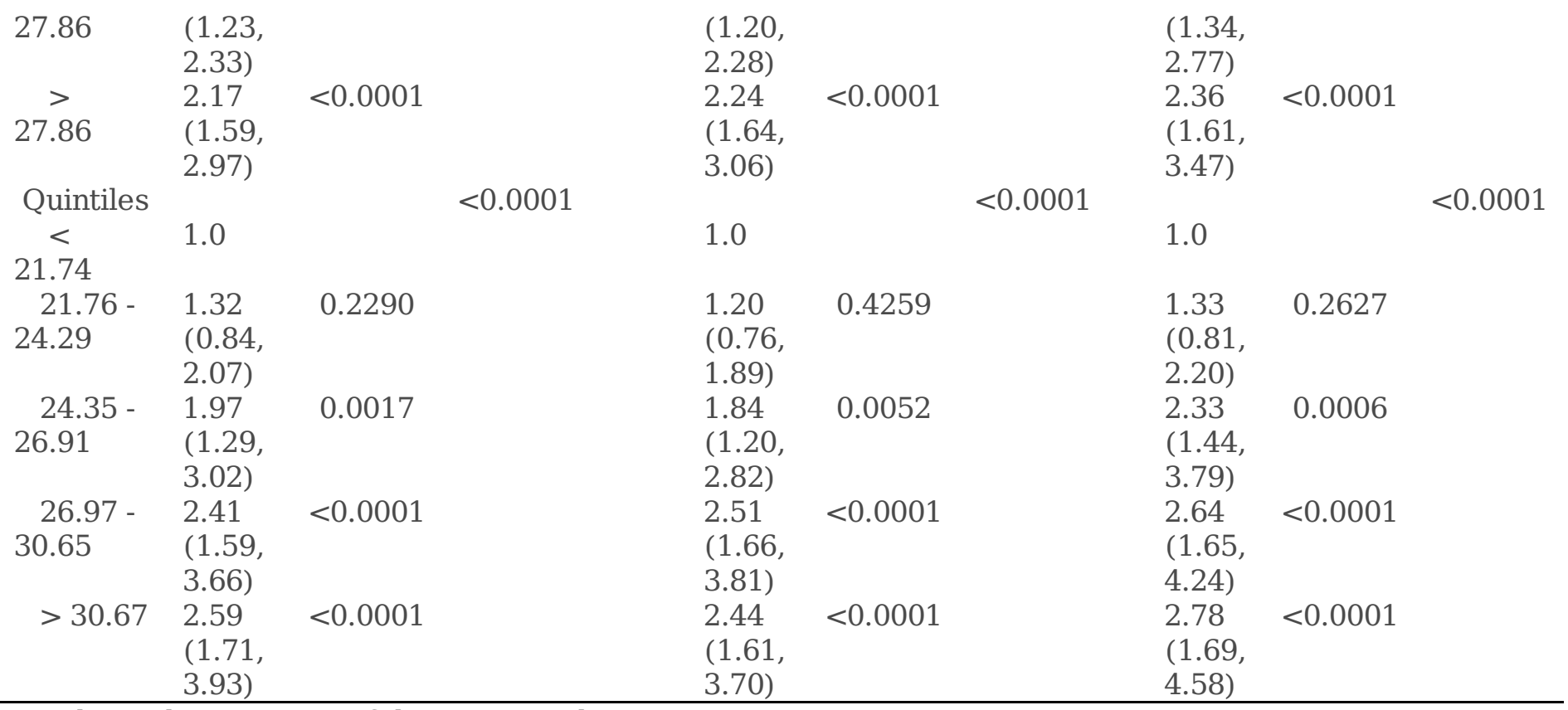

HR: hazard ratio; CI: confidence interval.

Models I and II were derived from Cox proportional hazards regression models: model I covariates were adjusted for age; gender; ethnicity; model II covariates were adjusted for age; gender; ethnicity; length of stay in ICU; HR; SBP; DBP; RR; SPO2; anion gap; serum bicarbonate; serum potassium; SCr; BUN; hematocrit; platelet count; WBC count; PTT; PT; INR; stroke; pneumonia; COPD; chronic liver disease; chronic renal disease; RRT; malignancy; vasoactive agent; SOFA; SAPSII. 
Table 3. Subgroup analysis of the associations between the NAR and 90-day mortality

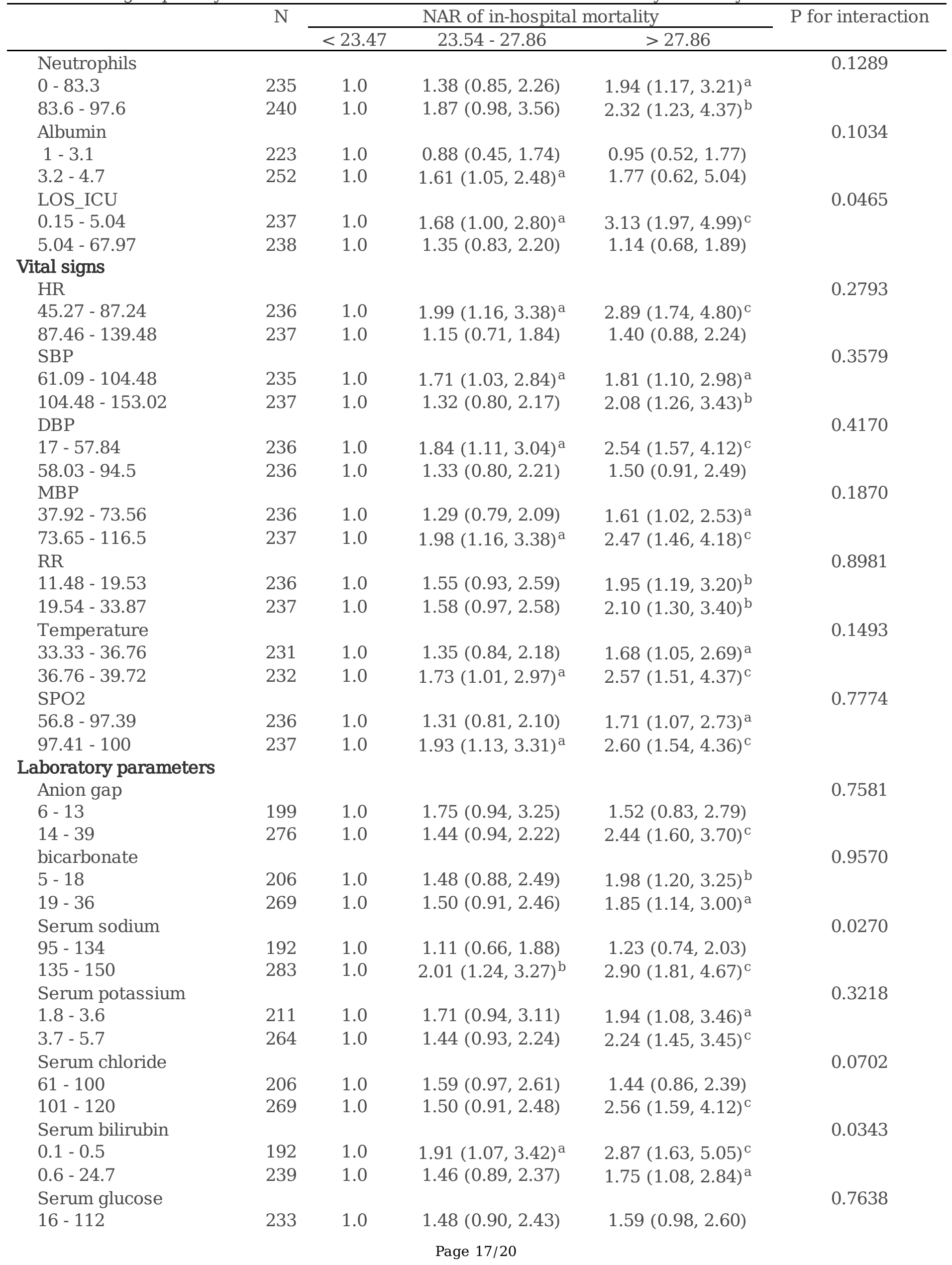




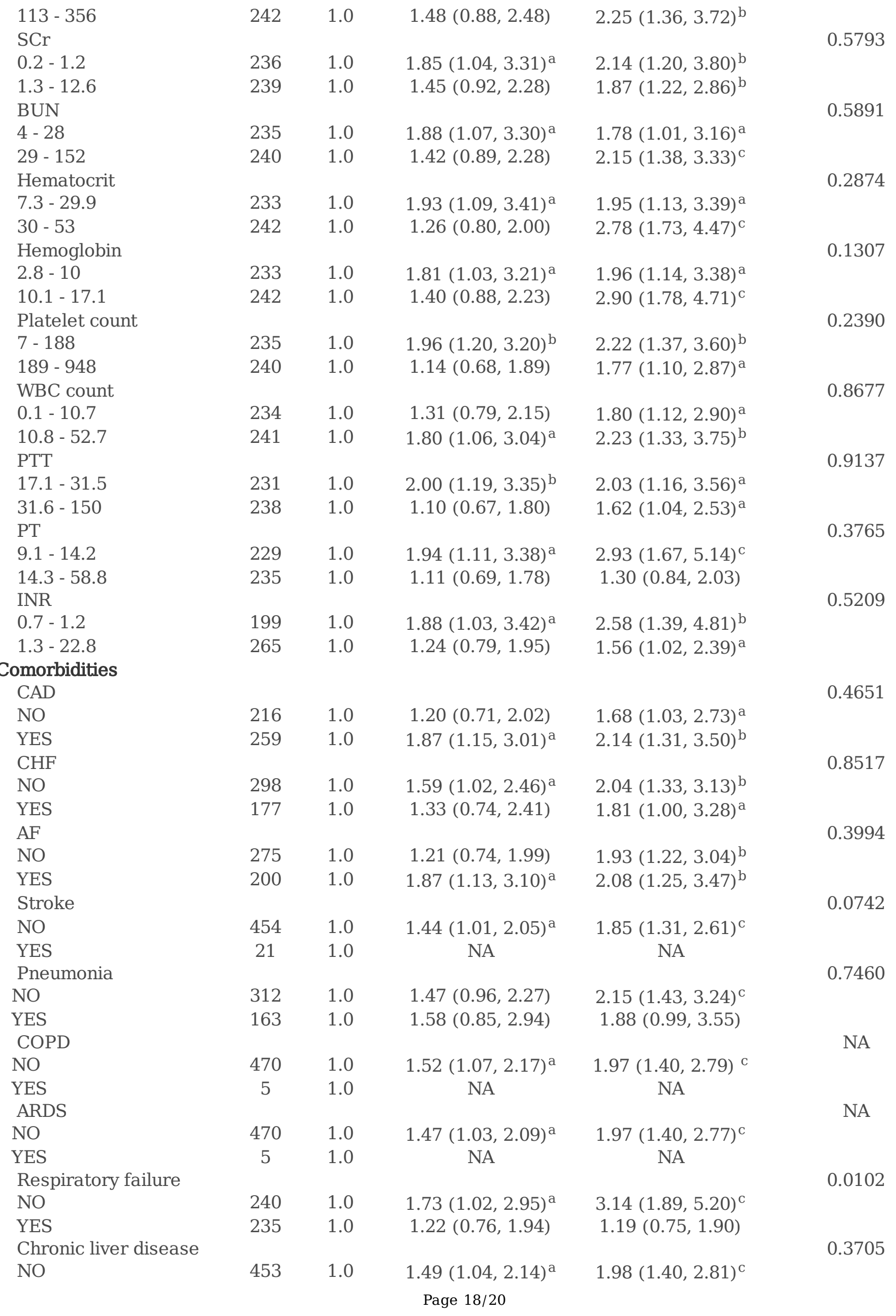




\begin{tabular}{|c|c|c|c|c|c|}
\hline YES & 22 & 1.0 & $0.50(0.09,2.75)$ & $3.26(0.66,16.14)$ & \\
\hline Chronic renal disease & & & & & 0.4300 \\
\hline NO & 375 & 1.0 & $1.69(1.11,2.57)^{\mathrm{a}}$ & $2.22(1.47,3.36)^{\mathrm{C}}$ & \\
\hline YES & 100 & 1.0 & $1.17(0.60,2.26)$ & $1.47(0.80,2.73)$ & \\
\hline RRT & & & & & 0.0044 \\
\hline NO & 402 & 1.0 & $1.42(0.96,2.11)$ & $2.29(1.58,3.32)^{\mathrm{C}}$ & \\
\hline YES & 73 & 1.0 & $0.87(0.36,2.09)$ & $0.45(0.18,1.11)$ & \\
\hline Malignancy & & & & & 0.3513 \\
\hline NO & 421 & 1.0 & $1.53(1.05,2.25)^{\mathrm{a}}$ & $2.15(1.48,3.12)^{\mathrm{C}}$ & \\
\hline YES & 54 & 1.0 & $1.58(0.59,4.26)$ & $1.08(0.41,2.85)$ & \\
\hline Vasoactive drug & & & & & 0.3256 \\
\hline $\mathrm{NO}$ & 110 & 1.0 & $0.83(0.35,1.96)$ & $1.78(0.87,3.64)$ & \\
\hline YES & 365 & 1.0 & $1.63(1.10,2.42)^{\mathrm{a}}$ & $2.02(1.36,2.98)^{\mathrm{C}}$ & \\
\hline \multicolumn{6}{|l|}{ Score systems } \\
\hline SOFA & & & & & 0.2866 \\
\hline $0-6$ & 216 & 1.0 & $1.66(0.94,2.96)$ & $2.47(1.37,4.46)^{b}$ & \\
\hline $7-21$ & 259 & 1.0 & $1.26(0.80,1.99)$ & $1.46(0.95,2.24)$ & \\
\hline SAPSII & & & & & 0.1969 \\
\hline $10-46$ & 231 & 1.0 & $1.56(0.86,2.82)$ & $3.01(1.67,5.42)^{\mathrm{C}}$ & \\
\hline $47-110$ & 244 & 1.0 & $1.49(0.95,2.34)$ & $1.41(0.92,2.15)$ & \\
\hline
\end{tabular}

NAR: neutrophil-albumin ratio; LOS_ICU: length of stay in intensive care unit; HR: heart rate; SBP: systolic blood pressure; DBP: diastolic blood pressure; MBP: mean blood pressure; RR: respiratory rate; SPO2: percutaneous oxygen saturation; BUN: blood urea nitrogen; SCr: Serum creatinine; WBC: white blood cell; PTT: partial thromboplastin time; PT: prothrombin time; INR: international normalized ratio; CAD: coronary heart disease; CHF: congestive heart failure; AF: atrial fibrillation; COPD: chronic obstructive pulmonary disease; ARDS: acute respiratory distress syndrome; RRT: renal replacement therapy; SOFA: Sequential Organ Failure Assessment; SAPSII: Simplified Acute Physiology Score.

Pvalue: ${ }^{\mathrm{a}} \mathrm{P}<0.05,{ }^{\mathrm{b}} \mathrm{P}<0.01,{ }^{\mathrm{c}} \mathrm{P}<0.001$

\section{Figures}
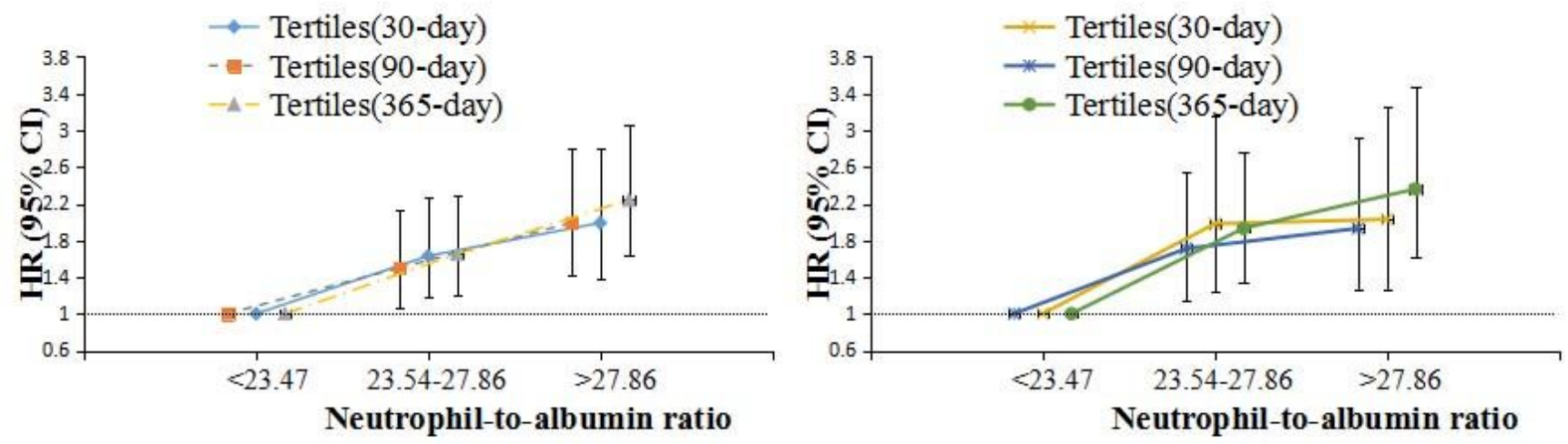

Figure. $1 \mathrm{HRs}$ ( $95 \% \mathrm{CIs}$ ) for mortality across tertile groups of NARs (Tertiles: model I and model II)

Figure 1 

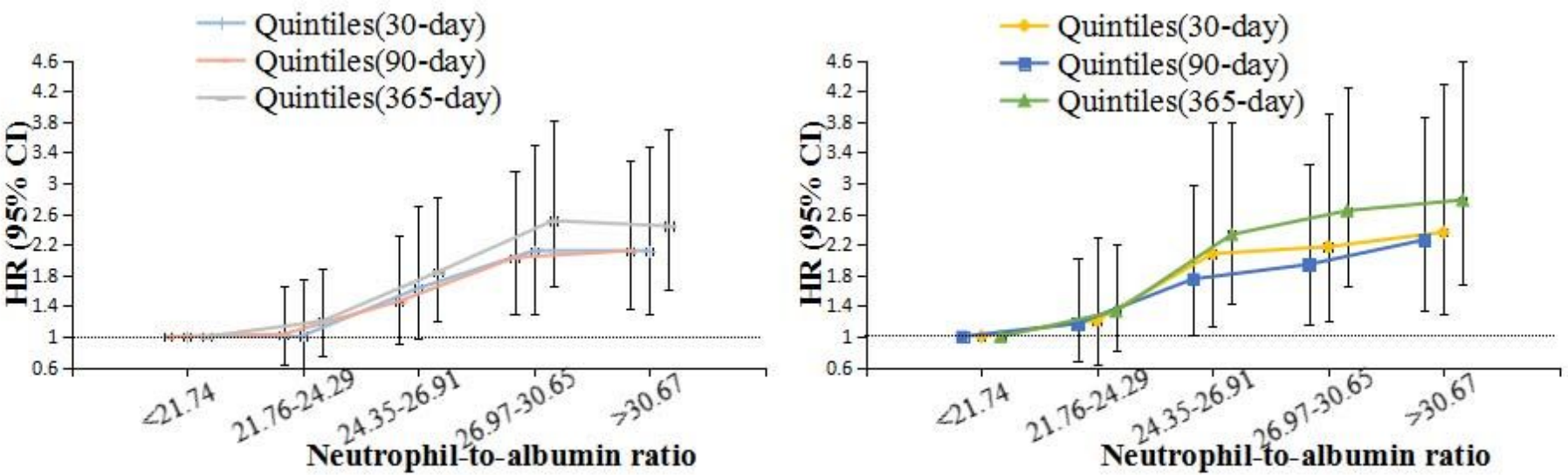

Figure. $2 \mathrm{HRs}$ ( $95 \% \mathrm{CIs}$ ) for mortality across quintile groups of NARs (Quintiles: model I and model II)

Figure 2 